\title{
Kommunikation und Versorgung in der Medizin
}

\author{
Welche Rolle spielt das Geschlecht?
}

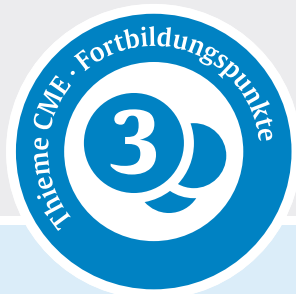

Julia Schreitmüller ${ }^{1,2}$, Bettina Pfleiderer ${ }^{1}$

1 Klinik für Radiologie und Medizinische Fakultät, Westfälische Wilhelms-Universität Münster

2 Institut für Arbeits-, Sozial-, und Umweltmedizin, Center for Health and Society, Universität Düsseldorf

ZUSAMMENFASSUNG

Soziale, aber auch biologische Geschlechteraspekte von Patienten sowie medizinischem Fachpersonal spielen eine wichtige Rolle im medizinischen Versorgungs- und Kommunikations- prozess. Studien bestätigen einen oft unbewussten „Gender Bias“, bei dem die Bedeutung von Geschlecht über- oder unterschätzt wird und damit Fehler während der Diagnose und Behandlung entstehen können. Patienten scheinen Symptome oft unterschiedlich wahrzunehmen und mitzuteilen. Aber auch Ärzte treffen zuweilen geschlechterabhängig unterschiedliche behandlungsrelevante Entscheidungen. Eine zunehmende Sensibilisierung des medizinischen Fachpersonals durch Forschung und Lehre, aber auch der breiten Öffentlichkeit ist dringend notwendig.

\section{Einführung}

Unsere Gesellschaft zeichnet sich durch Vielfalt im Hinblick auf Merkmale wie Geschlecht, Alter, Religionszugehörigkeit, sozioökonomischer Status und Migrationshintergrund aus und ist geprägt von ständiger Veränderung $[4,10]$. Beispielsweise kommt es aufgrund des demografischen Wandels zu einer alternden Gesellschaft und einer steigenden Zahl an Ein-Personen-Haushalten vor allem bei Frauen [7]. Aber auch die Bevölkerung mit Migrationshintergrund wird immer heterogener, da verstärkt Menschen aus Ländern nach Deutschland zuwandern, die dort bisher nur einen kleinen Teil ausgemacht haben [2]. Diversität bedeutet für den Gesundheitsbereich, dass unterschiedliche Versorgungsbedürfnisse von Patienten geäußert werden (z. B. hinsichtlich Aufklärung und Kommunikation) und aus medizinischer Sicht häufig ein unterschiedlicher Versorgungsbedarf bestehen kann (z. B. hinsichtlich medikamentöser oder psychosozialer Versorgung) [4]. Agierende im Gesundheitsbereich (Ärzte, Pflegepersonal, Psychologen, aber auch die Patienten selbst) werden dabei stetig vor neue Herausforderungen gestellt. Wird die Diversität der Gesellschaft nicht hinreichend in den Versorgungsprozess miteingebunden, bleiben Versorgungsbedürfnisse bzw. medizinischer Bedarf unerfüllt und nicht nur kommunikative Missverständnisse und Unzufriedenheit können die Folge sein, sondern auch gravierende negative Versorgungsergebnisse. Im Folgenden soll das Merkmal Geschlecht als wichtiger Aspekt einer diversitätssensiblen Versorgung in den Blick genommen werden. Besonders ist hier, dass nicht nur sozialgesellschaftliche, sondern auch biologische Unterschiede berücksichtig werden müssen, um eine adäquate Versorgung gewährleisten zu können. Zusätzlich muss erkannt werden, dass Geschlecht nicht als isolierter Faktor zu sehen ist, sondern immer auch zusammenhängt mit Merkma- len wie Kultur oder Alter. Beispielsweise ist das Östrogendefizit nach der Menopause bei Frauen im Gegensatz zu Männern einer der häufigsten Gründe für Gesundheitseinschränkungen im Alter (wie der Verlust an Knochendichte, kardiovaskuläre Erkrankungen, kognitive Störungen, Depression oder Inkontinenz) [18].

\section{Geschlechteraspekte in der Versorgung}

Epidemiologisch betrachtet machen zunächst die Unterschiede im Krankenstand und in der Inanspruchnahme medizinischer Leistungen zwischen Frauen und Männern den Geschlechterunterschied in der medizinischen Versorgung transparent. So waren im Jahr 2015 Frauen beispielsweise um 14\% häufiger krankgeschrieben als Männer, was unter anderem damit zusammenhängt, dass Frauen deutlich öfter Gesundheitsleistungen in Anspruch nehmen [25, 26, 33, 35] und häufiger aufgrund von psychischen Erkrankungen krankgeschrieben sind. Des Weiteren machen Schwangerschaftskomplikationen 73\% des Geschlechterunterschieds im Krankenstand aus und sind damit sicherlich als eine der Hauptursachen zu sehen. Mütter melden sich zudem eher als Väter krank, um ihr Kind bei Krankheit zu Hause zu versorgen [25].

Jedoch sind diese Unterschiede im Krankenstand nur ein (oberflächlicher) Aspekt unter vielen, in dem sich Frauen und Männer im Kontext von medizinischer Versorgung unterscheiden. So scheinen sie als Patient oder Patientin Gesundheit und Krankheit sowie Beschwerden und Symptome verschieden wahrzunehmen, sind oft nicht auf die gleiche Weise bereit und fähig sich diesbezüglich mitzuteilen und beschäftigen sich in unterschiedlichem Maße mit Gesundheit und Krankheit [9, 13]. Aber auch als Arzt nehmen sie Symptome manchmal anders wahr und treffen zu- 


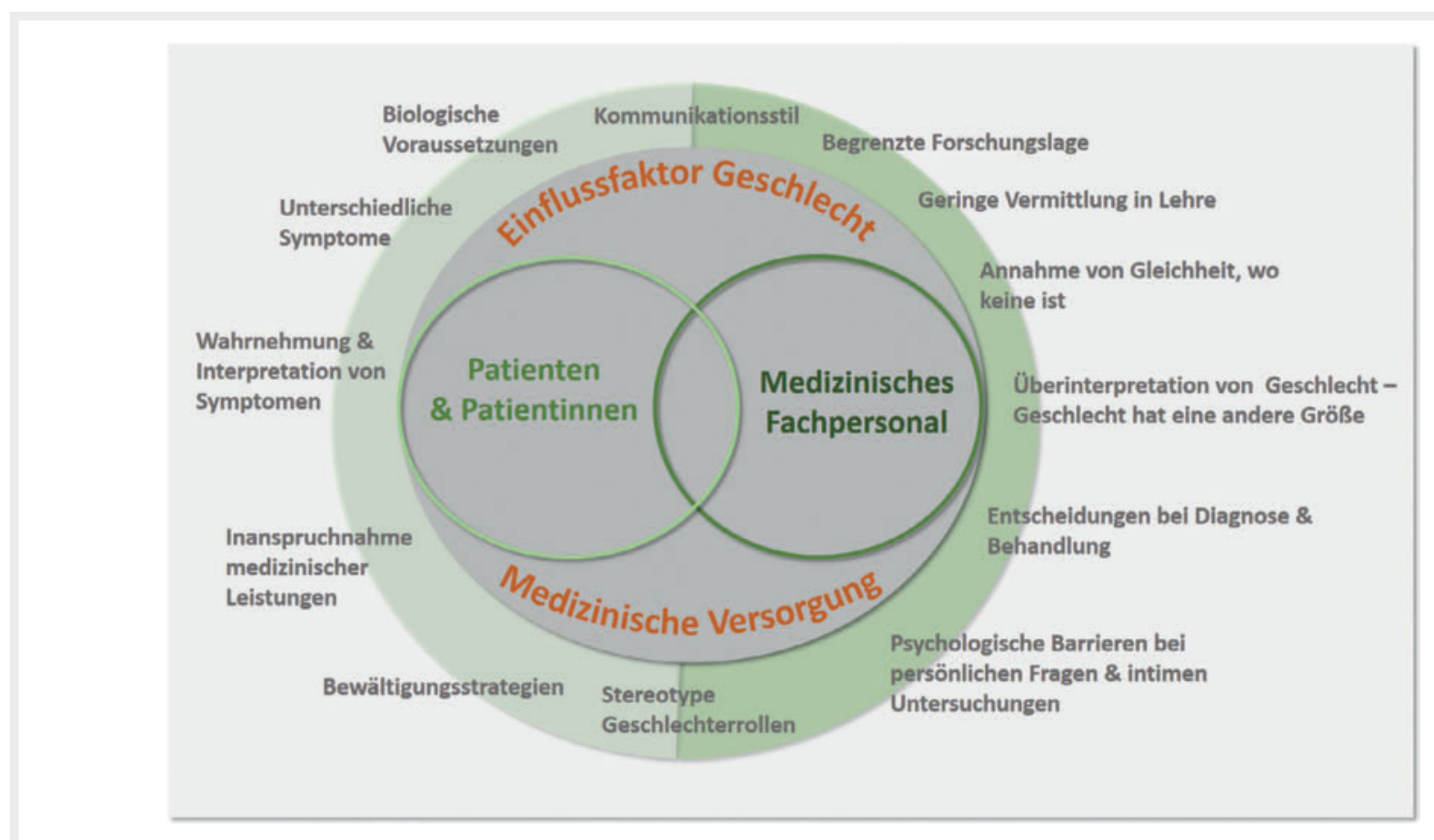

- Abb. 1 Mögliche geschlechterbezogene Einflussfaktoren auf die medizinische Versorgung (eigene Darstellung).

weilen unterschiedliche diagnostische und behandlungsrelevante Entscheidungen [17, 19]. Im Folgenden soll der Einfluss des Geschlechts in der medizinischen Versorgung genauer betrachtet und biologische, aber auch soziale Geschlechteraspekte aller Beteiligten (Patienten und medizinisches Fachpersonal) berücksichtigt werden, um sich der Komplexität dieses Themas nähern zu können ( $\mathbf{A} \mathbf{b b} \mathbf{b} \mathbf{1})$.

\section{Bedeutung des "Gender Bias“}

Medizinische Versorgung ist nicht geschlechterneutral. Dabei nimmt nicht nur das Geschlecht des Patienten Einfluss auf den Versorgungsprozess, sondern es kann auch entscheidend sein, ob das jeweilige Fachpersonal weiblich oder männlich ist. In diesem Zusammenhang bestätigen Studien einen oft unbewussten „Gender Bias“, der sich auf das Geschlecht der Patienten und Ärzte sowie auf das Geschlechterverhältnis in der Behandlungssituation bezieht und durch eine systematische Verzerrung zu Fehlern in der Behandlung führen kann. Beispielsweise ergab eine Studie zu Geschlecht und Diabetes, dass Patienten im Vergleich zu Patientinnen mit Typ-2-Diabetes signifikant seltener eine optimale Behandlung zur Vermeidung von möglichen Folgekomplikationen erhalten. Zudem wenden Ärztinnen bei Patientinnen und Patienten intensiver prognostisch wichtiges Präventionsmanagement als Ärzte an, und es gelingt ihnen besser als ihren männlichen Kollegen, Blutzuckerspiegel und Blutlipidspiegel zu senken [12].

Ursache des „Gender Bias“ kann zum einen eine Annahme von Gleichheit oder Ähnlichkeit zwischen den Geschlech- tern sein, obwohl relevante Unterschiede bestehen. Folge ist, dass Geschlechterunterschiede nicht berücksichtigt oder nicht als mögliche Erklärungsvariablen diskutiert werden. Zum anderen werden Geschlechterunterschiede angenommen, wo möglicherweise keine (bzw. weniger ausgeprägte) Unterschiede bestehen. Oder es findet eine Überbetonung der Variable Geschlecht im Vergleich zu anderen Faktoren (z. B. Alter oder Gewicht) statt, die nicht gerechtfertigt ist [16].

\section{Annahme von Gleichheit oder Ähnlichkeit - Medikamentöse Behandlung}

Ein Bereich, in dem Geschlechterunterschiede lange Zeit in der Forschung vernachlässigt worden sind und in der Praxis meist noch unberücksichtigt bleiben, ist die medikamentöse Behandlung. So wurden bis zum letzten Jahrhundert Frauen und weibliche Versuchstiere systematisch aus medikamentösen Forschungsbedingungen ausgeschlossen [27]. Grund war die Annahme, Studienergebnisse würden durch den weiblichen Hormonzyklus oder die Einnahme von Kontrazeptiva beeinflusst werden. Zudem befürchtete man einen frühzeitigen Drop-out aufgrund von Schwangerschaft während des Untersuchungszeitraums. Inzwischen besteht die Erkenntnis, dass genau diese hormonellen Einflüsse auf Medikamenteneinnahmen notwendig zu untersuchen sind. Genauso sollte das Risiko einer Medikamenteneinnahme während der Schwangerschaft konkreter erforscht werden, wobei zu bestimmten Medi- 
kamenten wie Antidepressiva bereits Studienergebnisse vorliegen [24].

Frauen und Männer unterscheiden sich auf vielfache Weise hinsichtlich des Gebrauchs und der Wirkung medikamentöser Behandlungen. Frauen befinden sich nicht nur häufiger in medikamentöser Behandlung, sondern leiden auch öfter als Männer unter deren Nebenwirkungen [26]. So besteht für Frauen im Vergleich zu Männern ein um $50 \%$ bis $70 \%$ höheres Risiko, dass Arzneimittelnebenwirkungen auftreten. Zudem kommt es bei Frauen vermehrt zu medikamentös induzierter Lebertoxizität, unerwünschten gastrointestinalen Ereignissen aufgrund von steroidaler entzündungshemmender Medikamente sowie zu medikamentös bedingten allergischen Hautausschlägen [31].

Obwohl die Pharmakokinetik (Effekte, denen ein Arzneimittel im Organismus unterliegt) neuer Substanzen in der Regel für Männer und Frauen separat beschrieben wird, werden die Behandlungseffekte (pharmakodynamische Effekte) fast nie geschlechtersensibel untersucht [41]. Der spezifische Effekt einer hohen Anzahl existierender Medikamente auf Frauen ist schlichtweg nicht bekannt. Die Regel ist, dass bei der medikamentösen Einnahme zwar zwischen Kindern und Erwachsenen unterschieden wird, aber nicht zwischen Männern und Frauen. Dabei gilt auch im Kontext der Arzneimittelwirkung, Geschlecht im Zusammenhang mit anderen Einflussfaktoren, z. B. Alter, zu sehen. Beispielsweise zeigt sich im Bereich der Psychopharmaka, dass prämenopausale Frauen besser als Männer auf selektive Serotoninwiederaufnahmehemmer (SSRI) ansprechen, wohingegen trizyklische Antidepressiva bei Männern und postmenopausalen Frauen ähnlich gut wirken [31].

Anders als auf gesellschaftlicher Ebene, setzen sich Forscher im Bereich der geschlechtersensiblen Medizin deshalb gegen eine generelle Gleichbehandlung von Frauen und Männern ein. Die Forderung nach einer Behandlung, die die unterschiedlichen biologischen Voraussetzungen zwischen den Geschlechtern anerkennt und berücksichtigt, beschränkt sich dabei nicht nur auf die Einnahme von Medikamenten, sondern bezieht sich auch auf Erkrankungen mit unterschiedlichen Prävalenzen, z. B. tritt Osteoporose deutlich häufiger bei Frauen auf. Eine zu starke Betonung dieser Geschlechterunterschiede birgt aber auch die Gefahr, dass Erkrankungen beim jeweils anderen Geschlecht nicht oder zu spät erkannt werden.

\section{Überbetonung der Variable Geschlecht - Beispiel psychosoziale Behandlung}

Eine solche Überbetonung der Variable Geschlecht findet häufig auf psychosozialer und psychiatrischer Ebene statt. Da deutlich mehr Frauen als Männer an psychischen Er- krankungen wie Angststörungen oder Depressionen leiden [5, 28], werden diese Erkrankungen bei Männern häufig übersehen oder zu spät erkannt. Deutlich wird das beispielsweise daran, dass Männer sich 3-mal so häufig wie Frauen suizidieren und bei $70 \%$ der Suizide eine depressive Erkrankung ursächlich ist [11]. Damit scheinen aktuell klare Defizite in Diagnostik und Versorgung depressiver Episoden (und psychischer Erkrankungen im Allgemeinen) vor allem bei Männern zu bestehen [40]. Ursächlich dafür könnte sein, dass zwar depressive Kernsymptome wie Traurigkeit, Niedergeschlagenheit und Anhedonie von beiden Geschlechtern etwa gleich häufig genannt werden, sich andere Symptome zwischen den Geschlechtern aber durchaus unterscheiden können. Zum Beispiel reagieren Männer bei einer Depression eher aggressiv und risikofreudig und greifen öfter zu Alkohol und Drogen. Diese externalen Symptome können besonders zu Beginn häufig die „klassisch“ internalen Symptome wie Selbstwertverlust, Antriebslosigkeit oder Verlust an Freude überdecken [40]. Vom Fachpersonal werden solche Verhaltensweisen dann häufig nicht als mögliche Depressionssymptome erkannt, sondern als „typisch männliche“ Abwehrstrategien“ eingeordnet [29].

Ein ähnliches Beispiel findet sich im Bereich der Essstörungen. Nur sehr wenige Erkrankungen weisen einen so enormen Geschlechterunterschied auf wie er bei Anorexia und Bulimia nervosa zu beobachten ist. Im Erwachsenenalter sind bis zu $90 \%$ der an Anorexie oder Bulimie Erkrankten weiblichen Geschlechts [15]. Das hat zur Folge, dass wenig Wissen zu diesen Erkrankungen bei Jungen und Männern besteht. So wurde Anorexie bei Männern lange Zeit nicht (an)erkannt und oft fälschlicherweise als Schizophrenie eingeordnet. Die symptomatische Körperschemastörung wurde dabei im Kontext eines wahnhaften Verhaltens verstanden. Das DSM-Kriterium einer mindestens seit 3 Monaten bestehenden Amenorrhö (Ausbleiben der Menstruation) konnte selbsterklärend nicht greifen. Im ICD-10 wurde dieses Kriterium deshalb durch das Vorhandensein einer endokrinen Störung (die sich bei Frauen als Amenorrhö und Libidoverlust und bei Männern als Libido- und Potenzverlust manifestiert) ersetzt [14].

Nicht nur auf psychosozialer Ebene, sondern auch in vielen anderen Bereichen werden bestimmte Diagnosen aufgrund stereotyper Zuordnung zu einem Geschlecht nicht oder verspätet gestellt. Beispielsweise wird ein Herzinfarkt [30] oder eine HIV-Erkrankung [6] bei Frauen viel später als bei Männern diagnostiziert. Grund dafür ist unter anderem, dass diese Erkrankungen als „untypisch“ für das weibliche Geschlecht eingeordnet werden und Frauen deshalb nicht als wahrscheinliche Kandidatinnen gelten. Während die Variable Geschlecht einerseits also stereotyp zu stark betont wird, sind paradoxerweise andererseits Geschlechterunterschiede in der Symptomatik dieser weitverbreiteten Erkrankungen oft nicht hinreichend bekannt. Zum Beispiel existiert eine deutliche Chancenun- 


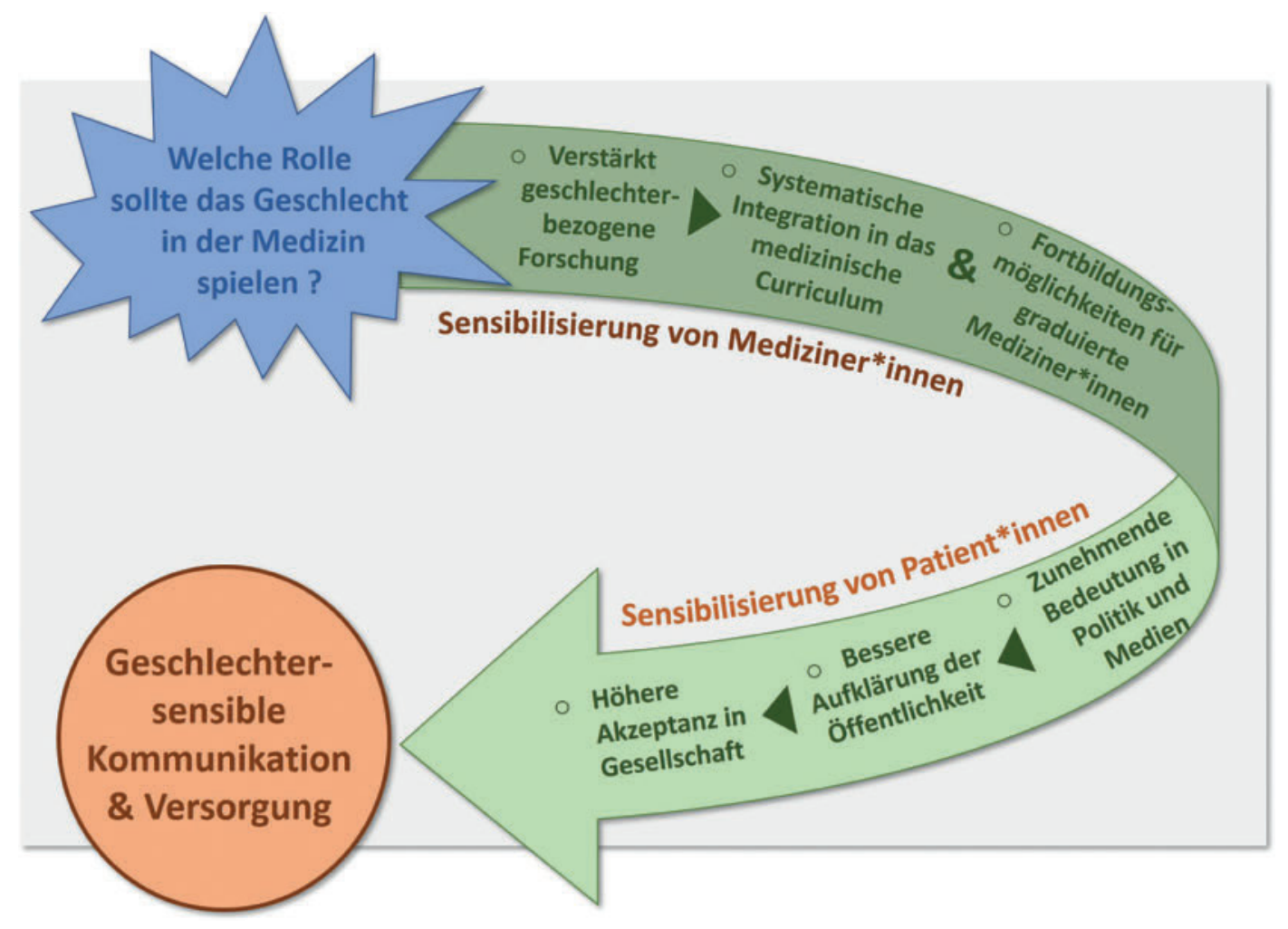

Abb. 2 Voraussetzungen auf dem Weg zu einer geschlechtersensiblen Versorgung (eigene Darstellung).

gleichheit von Männern und Frauen hinsichtlich kardiologischer Behandlungen. Da sowohl Patientinnen also auch behandelnde Ärzte häufig nicht mit einem Infarkt rechnen, erhalten Frauen oft nicht rechtzeitig eine adäquate Behandlung und kommen europaweit meist später ins Krankenhaus als Männer [32]. Grund hierfür kann ähnlich wie bei psychischen Erkrankungen sein, dass sich ein Herzinfarkt bei Frauen häufig symptomatisch anders äußert als bei Männern, z. B. durch Schmerzen der Kiefergelenke und des Rückens sowie vasovagale Beschwerden [34].

\section{Kommunikation und Interaktion}

Durch fortschreitende Technisierung der Medizin und zunehmenden Zeitdruck von medizinischem Fachpersonal tritt die Arzt-Patienten-Kommunikation als eigentlich zentraler Bestandteil des Behandlungsprozesses oft in den Hintergrund [21]. Dabei spielt bei Diagnostik und Behandlung, neben (fehlendem) geschlechterbezogenen Wissen, auch der Kommunikationsprozess zwischen Patienten und medizinischem Fachpersonal eine tragende Rolle. Vor allem der Hausarzt begleiten die Patienten häufig über viele Jahre und sind meist erste Anlauf- und Koordinationsstelle für die weitere medizinische Versorgung. Die Freiheit, das Geschlecht des Hausarztes selbst zu wählen scheint dabei besonders für Frauen entscheidend, wobei die Mehrheit (z. B. bei sexuellen Problemen) eine
Hausärztin bevorzugen würde. Diese geschlechtsspezifische Präferenz wird unter anderem mit einem patientenorientierten Kommunikationsstil erklärt, der häufiger von Ärztinnen praktiziert wird [17]. Während der Konsultation explorieren Ärztinnen die psychosozialen Umstände der Patienten durchschnittlich genauer, spenden dem emotionalen Zustand mehr Beachtung, treffen einen positiveren Ton, ermöglichen den Betroffenen eine Zusammenarbeit auf Augenhöhe und ermutigen zu mehr Teilhabe an medizinischen Entscheidungen. Ärzten wird dagegen häufig ein eher aufgabenorientierter Kommunikationsstil zugesprochen, der das Herausarbeiten der Krankheitsgeschichte sowie das Erklären von Diagnosen und präzisen Behandlungsstrategien beinhaltet [1].

Zudem treffen Ärzte zuweilen unterschiedliche Entscheidungen bezüglich Diagnose und Behandlung. Zum Beispiel führen Ärzte bei den gleichen Symptomen häufiger eine Rektaluntersuchung bei männlichen Patienten durch als ihre Kolleginnen. Dagegen nehmen Hausärztinnen im Vergleich zu Hausärzten bei Frauen eher eine Vaginaluntersuchung vor [20, 37]. Es scheinen psychologische Barrieren zu existieren, wenn es darum geht, Patienten des anderen Geschlechts sehr persönliche Fragen zu stellen oder intime Behandlungen vorzunehmen. Das führt zuweilen dazu, dass notwendige Behandlungen nicht stattfinden, Ärzte bezüglich dieser Untersuchun- 


\section{FAZIT}

In der medizinischen Versorgung und Forschung gilt es die Balance zu halten zwischen einer notwendigen Berücksichtigung des Einflussfaktors Geschlecht und dem Problem, Geschlechterunterschiede zu sehen, wo möglicherweise keine, oder deutlich kleinere als angenommen, bestehen (Überbetonung von Geschlecht). Beide Herangehensweisen können eine schlechtere Behandlung und damit negative gesundheitliche Konsequenzen für Patienten bedeuten. So führt zum Beispiel die Annahme von Gleichheit in der medikamentösen Behandlung bei Frauen zu einem deutlich erhöhten Risiko für Nebenwirkungen [8, 26]. Die Überbetonung von Geschlecht kann diagnostische Fehlentscheidungen zur Folge haben, da bestimmte Erkrankungen stereotyp einem Geschlecht zugeordnet werden. Nicht nur auf psychosozialer Ebene, z. B. werden Depressionen bei Männern häufiger übersehen, sondern auch in vielen anderen Bereichen, z. B. bei kardiovaskulären Erkrankungen, werden bestimmte Diagnosen deshalb nicht oder verspätet gestellt. Lösung auf dem Weg zu einer geschlechtersensiblen Versorgung und der Vermeidung von diesbezüglichen Fehlentscheidungen ist sicherlich eine deutliche Sensibilisierung des medizinischen Fachpersonals, aber auch der Patienten ( A Abb. 2). Diese Sensibilisierung erfolgt bereits in ersten Ansätzen durch geschlechterbezogene Forschung (z. B. muss Geschlecht bei Förderungen durch die EU in Studien berücksichtigt werden) und Lehre (z. B. bietet die Universität Halle-Wittenberg Handreichungen für die Integration geschlechtssensibler Medizin in die Lehre an, auch haben einige Universitäten bereits ein entsprechendes Wahlfach eingeführt [22, 23]) sowie entsprechende Fortbildungsmöglichkeiten (z. B. über die Deutsche Gesellschaft für Geschlechtsspezifische Medizin e. V.). Um medizinische Versorgung ausreichend verbessern zu können, sind jedoch weitere Bemühungen dringend notwendig, z. B. bedarf es einer systematischen Integration von geschlechterbezogenen Themen in das medizinische Curriculum [22, 36], was nach internationalen Bewertungsmaßstäben [39] bisher nur eine medizinische Fakultät in Deutschland erreicht hat.

Erst wenn sich die medizinische Ausbildung diesbezüglich ändert, wird die geschlechtersensible Medizin ihr Nischendasein in Forschung und Praxis verlassen können. Für zukünftige Ärzte sollte es als selbstverständlich gelten, dass die Berücksichtigung von sozialem und biologischem Geschlecht ein Teil der personalisierten, zielgerichteten Diagnostik und Therapie sein muss, um eine angemessene Behandlung für Frauen und Männer gewährleisten zu können und die Qualität in der Medizin zu erhöhen. Darüber hinaus sollte zukünftig auch eine zunehmende Sensibilisierung dahingehend stattfinden, dass Kommunikation und Behandlung im Versorgungsalltag immer mehr in einem „Cross-Cultural Setting“ stattfinden und somit nicht nur von biologischen und sozialen Geschlechteraspekten geprägt sind, sondern z. B. auch vor sprachlichen und kulturellen Herausforderungen aufgrund migrationsspezifischer Aspekte stehen [3]. Die Wechselwirkung von Geschlecht mit anderen sozialen Determinanten sollte demnach noch stärker Teil der geschlechtersensiblen Medizin werden. gen weniger erfahren sind und seltener relevante Befunde gemacht werden können. Auch ergeben Studien, dass Hausärzte und Internisten häufiger und in höheren Dosen Psychopharmaka, Sedativa und Analgetika als ihre Kolleginnen verschreiben (und Patientinnen diese häufiger verschrieben bekommen als Patienten) [19, 38]. Ärzte interpretieren Gesundheitsbeschwerden bei Frauen öfter als psychosomatisch als bei männlichen Patienten. Sie verschreiben zudem Frauen in der Menopause häufiger hormonelle Ersatztherapien und HIV-positiven Patienten häufiger Proteaseinhibitoren als dies bei Ärztinnen zu beobachten ist. Aber nicht nur das Geschlecht, sondern auch die aktuelle Lebensphase kann Einfluss auf medizinische Entscheidungen ausüben. Zum Beispiel verschreiben Hausärztinnen mit menopausalen Beschwerden Patientinnen im Klimakterium deutlich häufiger hormonelle Ersatztherapien als ihre männlichen Kollegen oder jüngeren Kolleginnen [1].

Darüber hinaus können auch soziale Geschlechtermerkmale der zu behandelnden Person Einfluss auf den versorgungsrelevanten Kommunikationsprozess nehmen. So unterscheiden sich Patienten oft in ihrer Einschätzung hinsichtlich Gesundheit und Krankheit und präsentieren bzw. erklären ihre Symptome in unterschiedlicher Weise oder versäumen dies. Zum Beispiel sind Männer mehr als Frauen geneigt dazu, gesundheitliche Beschwerden einschließlich psychischer Probleme zu verleugnen oder eigene Lösungsversuche zu finden. Frauen berichten dagegen früher und häufiger von gesundheitlichen Problemen [1]. Auch können Geschlechterunterschiede bezüglich der Bewältigung von gesundheitsbezogenen und anderen Problemen beobachtet werden. Wie beschrieben, nehmen Frauen beispielsweise deutlich häufiger professionelle Hilfe in Anspruch. Unklar bleibt dabei, ob Frauen zuweilen „übermäßigen“ Gebrauch von Gesundheitsangeboten machen (Frauen verursachen höhere Gesundheitskosten) oder ob Männer diese „ungenügend“ nutzen. Klar ist allerdings, dass die deutlich selteneren präventiven Arztbesuche auf Seiten der Männer negative Folgen aufweisen. Beispielsweise gehen rund $37 \%$ der Frauen, aber nur $23 \%$ der Männer in Deutschland zur Krebsfrühdiagnostik [32]. Die Einführung geschlechtersensibler Präventionsprogramme scheint dringend notwendig, um die Zielgruppe der Männer erreichen zu können. Unter anderen erweisen sich die in medizinischen Praxen ausgelegten Informationen über Vorsorgeuntersuchungen oft als problematisch. Aufgrund von sprachlichen Formulierungen und Bildmaterial scheinen sich Männer häufig nicht angesprochen zu fühlen. Notwendig sind offenbar andere Anreize, um Männer für ihre Gesundheit stärker zu sensibilisieren. Letztlich gilt es für Praktizierende im Gesundheitssystem zu berücksichtigen, auf welche Weise Frauen und Männer physische und psychische Beschwerden wahrnehmen, interpretieren und präsentieren. Dementsprechend lässt sich das medizinische Verhalten so ausrichten, dass die erforderliche individuelle Betreuung gewährleistet werden kann [1]. 
Interessenkonflikt

Erklärung zu finanziellen Interessen

Forschungsförderung erhalten: nein; Honorar/geldwerten Vorteil für Referententätigkeit erhalten: nein; Bezahlter Berater/interner Schulungsreferent/Gehaltsempfänger: nein; Patent/Geschäftsanteile/Aktien (Autor/Partner, Ehepartner, Kinder) an Firma (Nicht-Sponsor der Veranstaltung): nein; Patent/Geschäftsanteile/Aktien (Autor/Partner, Ehepartner, Kinder) an Firma (Sponsor der Veranstaltung): nein.

Erklärung zu nicht finanziellen Interessen

Die Autorinnen geben an, dass kein Interessenkonflikt besteht.

\section{Korrespondenzadresse}

Prof. Dr. med. Dr. rer. nat. Bettina Pfleiderer

Arbeitsgruppe Cognition \& Gender

Klinik für Radiologie

Albert-Schweitzer-Campus 1, Gebäude A16

48149 Münster

pfleide@uni-muenster.de

\section{Literatur}

[1] Berg M, Appelman Y, Bekker M (Hrsg.) Gender and Health Knowledge Agenda. Den Haag: ZonMW; 2015

[2] Binder-Fritz C, Rieder A. Zur Verflechtung von Geschlecht, sozioökonomischem Status und Ethnizität im Kontext von Gesundheit und Migration. Bundesgesundheitsblatt Gesundheitsforschung Gesundheitsschutz 2014; 57: 1031-1037

[3] Brzoska P, Razum O. Herausforderungen einer diversitätssensiblen Versorgung in der medizinischen Rehabilitation. Die Rehabilitation 2017; 56: 299-304

[4] Busch MA, Maske UE, Ryl L et al. Prävalenz von depressiver Symptomatik und diagnostizierter Depression bei Erwachsenen in Deutschland: Ergebnisse der Studie zur Gesundheit Erwachsener in Deutschland (DEGS1). Bundesgesundheitsblatt Gesundheitsforschung Gesundheitsschutz 2013; 56: 733-739

[5] del Castillo LS, Ruiz-Pérez I, de Labry Lima AO. Biological, psychosocial, therapeutic and quality of life inequalities between HIV-positive men and women-a review from a gender perspective. AIDS Rev 2010; 12: 113-120

[6] Demografischer Wandel in Deutschland. Heft 1: Bevölkerungs- und Haushaltsentwicklung im Bund und in den Ländern. Wiesbaden: Statistisches Bundesamt; 2011

[7] Drici M-D, Clément N. Is gender a risk factor for adverse drug reactions? Drug saf 2001; 24: 575-585

[8] Ette A, Mundil-Schwarz R, Sauer L et al. Ein neues Bild der Migration: Sozioökonomische Struktur und Arbeitsmarktintegration von Neuzuwanderern aus Drittstaaten in Deutschland. Bevölkerungsforschung Aktuell 2013; 2-11

[9] Faltermaier T, Hübner IM. Psychosoziale Gesundheitstheorien aus Geschlechterperspektive. In: Kolip P, Hurrelmann K, Hrsg. Handbuch Geschlecht und Gesundheit. Männer und Frauen im Vergleich. Bern: Hogrefe; 2016: 45-57
[10] Gardenswartz L, Rowe A. Managing diversity: A complete desk reference and planning guide. New York: McGraw Hill Professional; 1998

[11] Gößwald A, Lange M, Kamtsiuris P et al. DEGS: Studie zur Gesundheit Erwachsener in Deutschland. Bundesgesundheitsblatt Gesundheitsforschung Gesundheitsschutz 2012; 55: 775-780

[12] Gouni-Berthold I, Berthold HK, Mantzoros CS et al. Sex disparities in the treatment and control of cardiovascular risk factors in type 2 diabetes. Diabetes Care 2008; 31: 1389-1391

[13] Hagen C, Starker A. Epidemiologie - was fällt auf? Unterschiede in der Gesundheit von Frauen und Männern. Public Health Forum 2011; 2: 4-5

[14] Herpertz S, Zwaan M de, Zipfel S (Hrsg.) Handbuch Essstörungen und Adipositas. Heidelberg: Springer; 2015

[15] Jacobi F, Höfler M, Strehle J et al. Psychische Störungen in der Allgemeinbevölkerung. Nervenarzt 2014; 85: 77-87

[16] Jahn I. Methodische Probleme einer geschlechtergerechten Gesundheitsforschung. In: Kolip P, Hurrelmann K (Hrsg.) Handbuch Geschlecht und Gesundheit. Männer und Frauen im Vergleich. Bern: Hogrefe; 2016: 71-86

[17] Janssen SM, Lagro-Janssen ALM. Physician's gender, communication style, patient preferences and patient satisfaction in gynecology and obstetrics: a systematic review. Patient Educ Couns 2012; 89: 221-226

[18] Jaspers L, Daan NMP, van Dijk GM et al. Health in middleaged and elderly women: a conceptual framework for healthy menopause. Maturitas 2015; 81: 93-98

[19] Johnell K, Fastbom J. Gender and use of hypnotics or sedatives in old age: a nationwide register-based study. Int J Clin Pharm 2011; 33: 788-93

[20] Lagro-Janssen AL. Medicine is not gender-neutral: influence of physician sex on medical care. Ned Tijdschr Geneeskd 2008; 152: 1141-1145

[21] Löning P, Rehbein J (Hrsg.). Arzt-Patienten-Kommunikation: Analysen zu interdisziplinären Problemen des medizinischen Diskurses. Berlin New York: de Gruyter; 2011

[22] Ludwig S, Dettmer S, Peters H et al. Geschlechtsspezifische Medizin in der Lehre - noch in den Kinderschuhen. Dtsch Ärztebl 2016; 113: 2364-2366

[23] Ludwig S, Oertelt-Prigione $S$, Kurmeyer $C$ et al. A successful strategy to integrate sex and gender medicine into a newly developed medical curriculum. J Womens Health 2015; 24: 996-1005

[24] Macklin R. Enrolling pregnant women in biomedical research. Lancet 2010; 375: 632-633

[25] Marschall J, Nolting HD, Hildebrandt-Heene S et al. Gesundheitsreport 2016: Analyse der Arbeitsunfähigkeitsdaten. Schwerpunkt: Gender und Gesundheit: DAK-Gesundheit; 2016

[26] Merens A, van den Brakel M, Hrsg. Emancipation Monitor 2014. Den Haag: Netherlands Institute for Social Research and Statistics; 2014

[27] Merkatz RB, Junod SW. Historical background of changes in FDA policy on the study and evaluation of drugs in women. Acad Med 1994; 69: 703-707

[28] Möller-Leimkühler AM. Geschlecht und psychische Störungen. Public Health Forum 2011; 2: 5-7

[29] Möller-Leimkühler AM, Paulus N-C, Heller J. „Male depression" in einer Bevölkerungsstichprobe junger Männer. Risiko und Symptome. Nervenarzt 2007; 78: 641-50 
[30] Mosca L, Banka CL, Benjamin EJ et al. Evidence-based guidelines for cardiovascular disease prevention in women: 2007 update. J Am Coll Cardiol 2007; 49: 1230-1250

[31] Nieber K. Genderaspekte in der Pharmakotherapie. Zeitschrift für Komplementärmedizin 2014; 6: 17-23

[32] Prütz F. Rommel A. Inanspruchnahme ambulanter ärztlicher Versorgung in Deutschland. Journal of Health Monitoring 2017: 2: 88-94

[33] Regitz-Zagrosek V. Geschlecht und Herz-Kreislauf-Erkrankungen. Warum wir Gendermedizin brauchen. Internist 2017; 58: 336-343

[34] Saner H. Manifestation und Verläufe der koronaren Herzkrankheit bei Männern und Frauen - Konsequenzen für Diagnose und Therapie. Ther Umsch 2007; 64: 305-310

[35] Saß AC (Hrsg.). Gesundheit in Deutschland. Berlin: RobertKoch-Institut; 2015

[36] Schreitmüller J, Becker JC, Zsebedits D et al. Development and initial Experience of an online Exchange Platform on Sex and Gender Aspects in Medicine: "GenderMed-Wiki". GMS J Med Educ 2018; 35

[37] Shires DA, Stange KC, Divine G et al. Prioritization of evidence-based preventive health services during periodic health examinations. Am J Prev Med 2012; 42: 164-173
[38] van der Waals FW, Mohrs ], et al. Sex differences among recipients of benzodiazepines in Dutch general practice. BM] 1993; 307: 363-366

[39] Verdonk P, Mans LJ, Lagro-Janssen AL. Integrating gender into a basic medical curriculum. Med Educ 2005; 39: $1118-1125$

[40] Weißbach L, Stiehler M. Männergesundheitsbericht 2013. Im Fokus: psychische Gesundheit. Bern: Hans-Huber; 2013

[41] Yang Y, Carlin AS, Faustino PJ et al. Participation of women in clinical trials for new drugs approved by the Food and Drug Administration in 2000-2002. J Womens Health 2009; 18: 303-310

Bibliografie

DOI https://doi.org/10.1055/a-1134-9009 Nervenheilkunde 2020; 39: 468-474 (c) Georg Thieme Verlag KG Stuttgart · New York ISSN 0722-1541 


\section{Punkte sammeln auf CM/F.thieme.de}

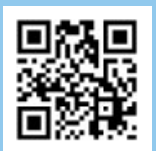

Diese Fortbildungseinheit ist bis zu 12 Monate online für die Teilnahme verfügbar.

Den genauen Einsendeschluss finden Sie beim Modul auf https://cme.thieme.de/CXERSIH.

Sollten Sie Fragen zur Online-Teilnahme haben, finden Sie unter https://cme.thieme.de/hilfe

eine ausführliche Anleitung. Wir wünschen viel Erfolg beim Beantworten

der Fragen!

Unter https://eref.thieme.de/CXERSIH oder über den QR-Code kommen Sie

direkt zum Artikel.

VNR 2760512020158722310

\section{Frage 1}

Welche Aussage zu Geschlechteraspekten in der medizinischen Versorgung trifft nicht zu?

A Geschlecht kann nicht als isolierter Faktor gesehen werden, sondern hängt immer auch mit Merkmalen wie Kultur oder Alter zusammen.

B Frauen sind deutlich häufiger krankgeschrieben als Männer (z. B. aufgrund von Schwangerschaftskomplikationen).

C Frauen und Männer melden sich inzwischen fast gleich häufig krank, um ihr Kind bei Krankheit zu Hause zu versorgen.

D Frauen und Männer beschäftigen sich in unterschiedlichem Maß mit Gesundheit und Krankheit.

E Biologische und soziale Geschlechteraspekte aller Beteiligten sollten im Behandlungsprozess berücksichtigt werden.

\section{Frage 2}

Welche Aussage trifft nicht auf den sogenannten Gender Bias zu?

A Sowohl das Geschlecht der Patienten als auch das des Fachpersonals kann Einfluss auf den Versorgungsprozess nehmen.

B Der Gender Bias kann eine Annahme von Gleichheit oder Ähnlichkeit zwischen den Geschlechtern sein, obwohl relevante Unterschiede bestehen.

C Folge des Gender Bias können schwerwiegende Fehler in der Behandlung sein.

D Eine zu starke Betonung von Geschlechterunterschieden birgt die Gefahr, dass Erkrankungen beim jeweils anderen Geschlecht zu spät erkannt werden.

E Der Gender Bias ist in der Regel nur im psychiatrischen und psychotherapeutischen Setting zu beobachten.

\section{Frage 3}

Welche Aussage zur medikamentösen Behandlung trifft nicht zu?

A Frauen haben im Vergleich zu Männern ein deutlich höheres Risiko, dass Arzneimittelnebenwirkungen auftreten.

B Bis zum letzten Jahrhundert wurden Frauen und weibliche Versuchstiere systematisch aus medikamentösen Forschungsbedingungen ausgeschlossen.

C Männer befinden sich häufiger als Frauen in medikamentöser Behandlung.

D Pharmakodynamische Effekte werden fast nie geschlechtersensibel untersucht.

E Die Pharmakokinetik neuer Substanzen wird in der Regel für Männer und Frauen separat beschrieben.

\section{Frage 4}

Welche Aussage zu psychosozialen Aspekten trifft zu?

A Aktuell scheinen keine Defizite in Diagnostik und Versorgung depressiver Episoden bei Männern zu bestehen.

B Männer zeigen häufig andere externale depressive Symptome als Frauen.

C Anorexie wurde bei Frauen lange Zeit nicht untersucht und häufig übersehen.

D Frauen suizidieren sich 3-mal so häufig wie Männer.

E Deutlich mehr Männer als Frauen leiden an Angststörungen und Depressionen.

\section{Frage 5}

Welche Aussage zur Überbetonung von Geschlecht trifft zu?

A Eine HIV-Erkrankung wird bei Männern durchschnittlich später diagnostiziert als bei Frauen .

B Es existiert keine deutliche Chancenungleichheit von Männern und Frauen hinsichtlich kardiologischer Behandlungen.

C Eine Überbetonung der Variable Geschlecht im Vergleich zu anderen Faktoren (z. B. Alter oder Gewicht) ist immer gerechtfertigt.

D Männer mit einem Herzinfarkt kommen europaweit meist später ins Krankenhaus als Frauen.

E Ein Herzinfarkt äußert sich bei Frauen häufig symptomatisch anders als bei Männern.

- Weitere Fragen auf der folgenden Seite ... 


\section{Frage 6}

Welche Aussage bezüglich Interaktion in der Behandlung trifft nicht zu?

A Hausärztinnen und Internistinnen verschreiben häufiger und in höheren Dosen Psychopharmaka, Sedativa und Analgetika als ihre männlichen Kollegen.

B Vor allem Frauen ist es wichtig, das Geschlecht des Hausarztes frei wählen zu können.

C Ärztinnen mit menopausalen Beschwerden verschreiben Patientinnen im Klimakterium öfters hormonale Ersatztherapien als ihre männlichen Kollegen.

D Während Ärzte häufiger eine notwendige Rektaluntersuchung bei männlichen Patienten durchführen, nehmen Ärztinnen eher eine indizierte Vaginaluntersuchung bei Frauen vor.

E Ärzte interpretieren Gesundheitsbeschwerden bei Frauen öfter als psychosomatisch als bei männlichen Patienten.

\section{Frage 7}

Welche Aussage zu sozialen Geschlechtermerkmalen der Patienten trifft nicht zu?

A Männer sind mehr als Frauen dazu geneigt, gesundheitliche Beschwerden zu verleugnen oder eigene Lösungsversuche zu finden.

B In Deutschland nehmen Männer deutlich häufiger als Frauen das Angebot zur Krebsfrühdiagnostik in Anspruch.

C Häufig nehmen Frauen und Männer gesundheitliche Beschwerden unterschiedlich wahr und interpretieren und präsentieren sie anders.

D Frauen verursachen höhere Gesundheitskosten als Männer.

E Frauen nehmen deutlich häufiger als Männer professionelle Hilfe in Anspruch.

\section{Frage 8}

Welcher Schritt zur Sensibilisierung des medizinischen Fachpersonals wurde in Deutschland bisher nicht gegangen?

A Eine systematische Integration von geschlechterbezogenen Themen in das medizinische Curriculum findet an den meisten medizinischen Fakultäten statt.

B Bei Förderungen durch die EU muss Geschlecht in Studien berücksichtigt werden.

C Einige Universitäten haben bereits ein entsprechendes Wahlfach eingeführt.

D Die Deutsche Gesellschaft für Geschlechtsspezifische Medizin e. V. bietet eine entsprechende Fortbildungsmöglichkeit an.

E Die Universität Halle-Wittenberg bietet Handreichungen für die Integration geschlechtssensibler Medizin in die Lehre an.

\section{Frage 9}

Welche Aussagen zur Kommunikation von Ärzten trifft nicht zu?

A Ärztinnen praktizieren häufiger einen patientenorientierten Kommunikationsstil.

B Ärztinnen fragen öfter nach psychosozialen Umständen ihrer Patienten als ihre männlichen Kollegen.

C Ärzte tragen dem emotionalen Zustand ihrer Patienten stärker Rechnung als Ärztinnen.

D Ärzte pflegen häufiger einen aufgabenorientierten Kommunikationsstil wie zum Beispiel das Erklären von Diagnosen oder präzisen Behandlungsstrategien.

E Ein patientenorientierter Kommunikationsstil kann Patienten ermutigen, mit ihren Behandlern auf Augenhöhe zu kommunizieren.

\section{Frage 10}

Soziale Geschlechtermerkmale von Patienten können Einfluss auf den behandlungsrelevanten Kommunikationsprozess nehmen. Welche Aussage trifft zu?

A Frauen nehmen seltener Präventionsangebote wahr bzw. sprechen mit ihrem Arzt seltener über Gesundheitsangebote.

B Frauen und Männer unterscheiden sich nicht in der Häufigkeit, bei ihrem Arzt gesundheitliche Probleme anzusprechen.

C Männer, im Vergleich zu Frauen, neigen dazu psychische Probleme zu verleugnen und eigene Lösungen zu finden.

D Die in Praxen ausliegenden Informationen zu Präventionsangeboten scheinen öfters Männer als Frauen zu motivieren diese auch wahrzunehmen.

E Ärzte neigen dazu, die Beschwerden von Patientinnen ernster zu nehmen als die von Patienten. 